Beim Erhitzen verpufft dieses Salz ziemlich kräftig und erinnert somit an das Knallgold, $\mathrm{Au} \mathrm{N}_{2} \mathrm{H}_{9} \mathrm{O}_{3}$, wobei unsere Analyse einwandfrei zeigt, daß die Goldstickstoffbindungen den explosiven Charakter tragen, ohne daß bei der Detonation eice Verbrennung stattfindet.

Verdünnte Salzsäure löst mit blaßgelber Färbung und läßt erst nach längerem Erhitzen mit Chlorbarium Bariumsulfat ausfallen, woraus folgt, daß ein echtes Amidosulfonat rorliegt.

In Wasser ist dieses Salz bei gewöhnlicher Temperatur sehr schwer, in der Hitze leichter löslich. Die gelbe Lösung ändert ibre Farbe durch Zusatz von Kalilauge oder Ammoniak nicht und liefert mit Formalin oder noch besser mit Tetraformal-trisazin einen festhaftenden Goldspiegel. Wasserstoffsuperoxyd wird lebhaft katalysiert unter gleichzeitiger Reduktion zu kolloidalem Gold, das lebhaft Farbenwechsel von gelbrot, braunrot und scbließlich grünen Nuancen zeigt.

222. Ad olf Kaufmann und Louis G. Vallette: Über eine neue Darstellung von Cyclamin-Aldehyden und -Alkoholen.

[Vorlăufige Mitteilung.]

(Eingegangen am 21. Mai 1912.)

Die Reaktionsfähigkeit der Methylgruppen in 2- und 4Stellung zum Stickstoffatom im Pyridinringe ist durch ibre Kondensationsfähigkeit mit Carbonylsauerstoff seit langem experimentell begründet und die Entstehung der $\mathrm{Pbthalone}$ und der $\mathrm{Cyanine}$ auf diese zurückgeführt worden 1). Weitere Beweise für die Beweglichkeit der Methylwasserstoffatome sind die Synthesen der Chiualdin- und Lepidin-oxalester durch Wilhelm Wislicenus und E. Kleisinger'), ebenso wie die Untersuchungen von E. Vongerichten und Rotta ${ }^{3}$ ) über die Benzoylierungsprodukte der Chinaldin-isobase ${ }^{4}$ ).

1) Jacobson und Reimer, B. 16, 513, 1082, 2602 [1883]; M. C. Traube, B. 16, 297, 878 [1883]; O. Wallach und M. Wüsten, B. 16, 2057 [1883]; Einhorn, B. 18, 3465 [1885]; Einhorn und Liebrecht, B. 20, 1592 [1887]; v. Miller und Spady, B. 18, 3402 [1885]; W. Königs, B. 32, 223 [1899]; Eibner und Lange, A. 315, 308.

2) B. 42, 1140 [1909]; siehe auch B. 30, 1479 [1897].

3) B. 44, $1419[1911]$.

7) und ncuerdings M. Scholtz, Einwirkung von Essigsäureanhydrid auf $\alpha$-Picolin, B. 45, 734 [1912]. 
Nun baben bereits zablreiche Arbeiten gezeigt, daß außer in dem Kupplungsbestreben mit Aldebyden ganz allgemein Körper mit reaktionsfähiger Methyl- oder Methylengruppe im Verhalten gegen salpetrige Säure, Diazobenzol, Nitroso-dialkylanilinen usw. charakteristisch übereinstimmen. Speziell die letztere Reaktion, die zur Bildung von leicht hydrolytisch spaltbaren Azomethinen Veranlassung gibt, ist seit einigen Jabren für die Darstellung von vielen Aldehyden und Ketonen von großer Wichtigkeit geworden, wie dies F. Sachs und seine Schüler in einer Reibe von Abbandlungen gezeigt baben.

Die Untersuchungen, die der eine von uns schon vor längerer Zeit zu diesem Zwecke mit Chinaldin, Lepidin und ăhnlichen Basen einerseits und Nitroso-dimethylanilin usw. andererseits angestellt hat, haben ergeben, $\mathrm{da} \beta$ auch diese Reaktionen in normaler Weise zu den gesuchten Realktionsprodukten führen. Die Analogie dieser Basen mit den bekannteren reaktionsfähigen Methylverbindungen (Dinitrotoluol, Nitroparalfine) ist also eine allgemeine.

Die ersten Resultate dieser Arbeiten sind in einer Patentanmeldung ${ }^{1}$ ) niedergelegt, bisher aber sonst einer weiteren Veröffentlichung ans verschiedenen Gründen vorenthalten worden. Unterdessen haben die HHrn. A. Porai-Koschitz, A uschkap und Amsler') eine Mitteilung über denselben Gegè̀stand gemacht, und dies veranlaßt uns, auch unsererseits über die in vielfacher Hinsicht interessante Reaktion zu berichten.

Während $m s$-Methyl-acridin ganz glatt mit Nitrosodimethylanilin unter den verschiedensten Bedingungen reagiert, erfolgt die Kondensation mit $\mathrm{Cbinaldin,} \mathrm{Lepidin,} \alpha$-Picolin augenscheinlich überhaupt nicht. Es überraschte uns deshalb einigermaßen, als es sich berausstellte, daß die quartären $S a l z e$ dieser Basen mit großer Leichtigkeit in Reaktion treten.

Die Reaktionsfähigkeit des Methyls ist also durch die Absättigung des Stickstoffs bedeutend gesteigert worden, während sie sonst gerade auf die Ungesättigtkeit der Radikale zurückgeführt wird ${ }^{3}$ ). Nach D. Vorländert) ist nun aber auch der Ammoniumrest nicht gesättigt, sondern gleichfalls ungesättigt - wie aus der Doppel- und Komplex-Salzbildung gefolgert werden darf -, und er bat deshalb auch eine reaktionserleichternde Wirkung auf benachbarte Gruppen. Diese äußert er in dem Maße besonders kräftig, als seine direkte Außenwirkung auf Addenden gering ist.

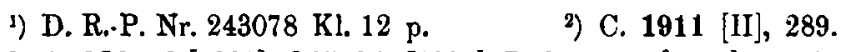

s) A. 320, 99 [1901]; 845, 251 [1906]; B. 34, 3617 [1901]; 38, 4142 [1902].

4) Gütige Priratmitteilung. 
So gibt z. B. Chinaldin-jodätbylat, mit Nitroso-dimethylanilin in Alkohol erwärmt, allmählich rotviolette Färbung. Viel rascher tritt der Farbumschlag bei Zusatz von einigen Tropfen Pi peridin ein. Das Kondensationsprodukt I wird durch Mineralsäuren

I.

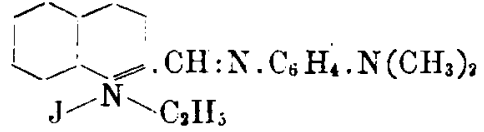

II.<smiles>CCCCNN=CC1CCCN1CC</smiles>

in $p$-Amino-dimethylanilin und das Jodätbylat des Chinolyl2-methauals gespalten, das durch Überfübrung in das entsprechencle Hydrazon Il genügend als Aldebyd charakterisiert ist.

Über den weiteren Abbau dieser Hydrazone durch Abspaltung von Halogenalkyl, sowie die Gewinnung der Aldebyde und Amine werden wir später berjchten. Insbesondere sollen das p-Methoxylepidin und das $\beta$-Kollidin der neuen Reaktion unterworfen werden.

Experimentelles.

Kondensation des Nitroso-dimethylanilins mit Methylverbindungen. $p$-Dimethylamino-anil des Chinolyl-2metbaual-jodäthylats (Formel I).

Chinaldin-jodätbylat ( $7.5 \mathrm{~g})$, Nitroso-dimethylanilin und einige Tropfen Piperidin wurden in $100 \mathrm{ccm}$ Alkobol aufgekocht. Die anfangs grüne Lösung nimmt nach wenigen Minuten rotviolette Färbung an. Nach dreistündigem Sieden am Rückflußkühler wurde zur Krystallisation gestellt. Die ausgeschiedenen grünen Nadeln (10 g) wurden mebrmals aus Alkobol umkrystallisiert. Die Substanz schmilzt unter Zersetzung gegen $200^{\circ}$ und ist sehr schwer verbrennlict.

0.1289 g Sbst.: 0.2600 g $\mathrm{CO}_{2}, 0.0658 \mathrm{~g} \mathrm{H} \mathrm{H}_{2} \mathrm{O} .-0.1132 \mathrm{~g}$ Sbst.: $10 \mathrm{ccm} \mathrm{N}$ $\left(19^{\circ}, 720 \mathrm{~mm}\right) .-0.2596 \mathrm{~g}$ Sbst.: $0.1412 \mathrm{~g} \mathrm{Ag} \mathrm{J}$.

$$
\begin{aligned}
& \mathrm{C}_{20} \mathrm{H}_{22} \mathrm{~N}_{3} \text { J. Ber. C 55.7, } \mathrm{H} \text { 5.1, N 9.7, J } 29.4 \text {. } \\
& \text { Gef. »55.0, » 5.7, » 9.1, 》 29.4. }
\end{aligned}
$$

Cbinolinjodätbylat in derselben Weise bebandelt gibt die Reaktion nicht. Es kondensiert also das Methyl des Chinaldins mit der Nitrosogruppe. Das Jodäthylat ist wenig löslich in kaltem Wasser und Alkohol, löst sich aber leicht in der Siedehitze. Die alkoholische Lösung ist violettrot, die wäßrige etwas rotstichiger.

Auch Nitroso-phenole kondensieren mit Chinaldinjodätbylat. Mit Nitroso-napbtbol erhält man eine intensiv olivgrüne Lösung, aus der sich nach längerem Stehen aber bloß ein barzartiger Niederschlag absetzte. Nitroso-antipyrin gibt ebenfalls ein Kondensationsprodukt. 
Jodalkylate des $p$-Dimetbylamino-anils des Pyridyl-2metbanals.

$\alpha-P i c o l i n$ reagiert mit Jodmetbyl mit großer Heftigkeit. Die gelbliche Reaktionsmasse krystallisiert aus heißem Alkohol in langen, vollkommen weißen Nadeln, die bei $224^{\circ}$ schmelzen. Das Jodmethylat kondensiert sich mit Nitrosodimetbylanilin bei Gegenwart von Piperidin schon in der Kälte, rascher bei der Siedetemperatur des Alkohols. Aus der roten Lösung scheidet sich beim Erkalten das Kondensationsprodukt in grünen Nädelchen ab. Die Substanz enthält ein Molekül Krystallalkohol, das im Vakuum und bei höherer Temperatur abgegeben wird; der Körper erscheint dann rot. Er ist schwer verbrennlich.

$0.1700 \mathrm{~g}$ Sbst.: $0.3121 \mathrm{~g} \mathrm{CO}_{2}, 0.0882 \mathrm{~g} \mathrm{H}_{2} \mathrm{O} .-0.1352 \mathrm{~g}$ Sbst.: $13.4 \mathrm{ccm}$ $\mathrm{N}\left(18^{0}, 720 \mathrm{~mm}\right) .-0.1650 \mathrm{~g}$ Sbst.: $0.0940 \mathrm{~g} \mathrm{AgJ}$.

$\mathrm{C}_{15} \mathrm{H}_{18} \mathrm{~N}_{3} \mathrm{~J}, \mathrm{C}_{3} \mathrm{H}_{5} .0 \mathrm{H}$. Ber. C 49.4, H 5.8, N 10.2, J 30.8 .

Gef. " 50.1 , " 5.8, > 11.0, 30.8 .

Das Derivat schmilzt bei ungefähr $185^{\circ}$ unter Zersetzung. Es ist in heißem Wasser und Alkohol leicht löslich, unlöslich in Äther, Benzol usw.

Picolin-jodätbylat, in analoger Weise mit Nitreso-dimethylanilin behandelt, liefert ein Kondensationsprodukt mit ähnlichen Eigenschaften. Grüne Nadeln, die beim Trocknen rot werden.

$0.1491 \mathrm{~g}$ Sbst.: $0.2748 \mathrm{~g} \mathrm{CO}_{2}, 0.0738 \mathrm{~g} \mathrm{H}_{2} \mathrm{O}$. $\mathrm{C}_{16} \mathrm{H}_{22} \mathrm{~N}_{3}$ J. Ber. C 50.1, H 5.7.

Gef. \50.3, 5.5 .

Scbließlich gibt das Lepidin-jodmetbylat mit Nitroso-dimethylanilin einen kupferglänzenden Körper, der sich in Alkohol mit prachtvoller blauer Farbe löst. Es tritt also hier derselbe Farbunterschied zwischen Lepidin- und Cbinaldin-Derivaten auf, der schon bei den Cyaninen beobachtet wurde.

\section{Kóndensation von}

Nitroso-dimetbylanilin mit ms.-Methyl-acridin.

Schmilzt man äquimolekulare Mengen von $p$-Nitroso-dimethylanilin und Methylacridin zusammen, so tritt bei $100-120^{\circ}$ lebhafte Wasserentwicklung ein, und die anfänglich grüne Flüssigkeit erstarrt allmählich zu einem rotbraunen Krystallkuchen. Die neue Verbindung ist in den üblichen Lösungsmitteln äußerst schwer löslich. Durch Estraktion mit Alkohol wird sie von nicht in Reaktion getretenen A usgangsmaterialien und gegebenenfalls Verunreinigungen befreit und stellt dann ein orangerotes Pulver dar. Kocht man dieses mit sehr viel Alkohol aus, sc gewinnt man grobe Krystalle rom Schmp. $234^{\circ}$, 
während aus den Mutterlaugen feine Schuppen erhalten werden, die bei $210-211^{\circ}$ schmelzen. Beide Produkte besitzen nach den Analysen die Zusammensetzung $\mathrm{C}_{23} \mathrm{H}_{19} \mathrm{~N}_{2} . \mathrm{H}_{2} \mathrm{O}$.

$0.1321 \mathrm{~g}$ Sbst.: $0.3741 \mathrm{~g} \mathrm{CO}_{2}, 0.0705 \mathrm{~g} \mathrm{H}_{2} \mathrm{O}$. $-0.1144 \mathrm{~g}$ Sbst.: $0.3254 \mathrm{~g}$ $\mathrm{CO}_{2}, 0.0644 \mathrm{~g} \mathrm{H}_{2} \mathrm{O}$ - $-0.1534 \mathrm{~g}$ Sbst.: $17.1 \mathrm{ccm} \mathrm{N}\left(18^{\circ}, 716 \mathrm{~mm}\right)$.

$$
\begin{aligned}
& \mathrm{C}_{22} \mathrm{H}_{19} \mathrm{~N}_{2}, \mathrm{H}_{2} \mathrm{O} \text {. Ber. C 77.0, H 6.1, N } 12.2 \text {. } \\
& \text { Gef. 》 77.2, 77.5, 》5.9, 6.2, 》12.1. }
\end{aligned}
$$

Der in Alkohol unlosliche bedeutend größere Teil schmilzt dagegen bei ca. $244^{\circ}$. Ein Produkt mit demselben Schmelzpunkt entsteht, wenn der unten beschriebene Acridyl-aldehyd mit $p$-Aminodimethylanilin zusammengeschmolzen wird. Die Kondensation geht sofort unter Selbsterwärmung vonstatten.

Hydrolytische Spaltung der Kondensations. produkte durch Mineralsäuren.

$$
\text { Acridyl-9.methanal, }
$$

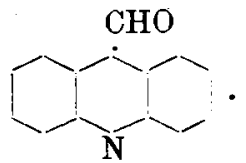

Das orangerote Kondensationsprodukt des Metbyl-acridins mit Nitroso-dimethylanilin löst sich in verdünnter Salzsäure augenblicklich in der Kälte mit gelber, grün fluorescierender Farbe auf. Ist die Lösung konzentriert genug, so erfolgt auch sofort die Ausscheidung von gelben Nadeln. Sie sind das Cblorhydrat des Acridyl-aldebyds, der durch Soda als voluminöser Brei niedergeschlagen wird. Der Aldehyd ist bereits von Bernthsen und Muhlert') durch Oxydation der Acridyl-acrylsäure dargestellt worden. Er schmilzt nach mehrfacher Reinigung durch Krystallisation nach unseren Beobachtungen bei $148^{\circ}$.

$0.11610 \mathrm{~g}$ Sbst.: $10.8 \mathrm{ccm} \mathrm{N}\left(19^{0}, 710 \mathrm{~mm}\right)$.

$\mathrm{C}_{14} \mathrm{H}_{9}$ NO. Ber. N 6.7. Gef. N 6.9.

Der Aldehyd gibt mit Phenylhydrazin in salzsaurer Lösung einen schönen violetten Farbstoff. Zur Charakterisierung wurden noch das Anil und das Oxim dargestellt.

Das Anil bildet sich schon bei gewöhnlicher Temperatur unter Selbst. erwärmung aus den beiden Komponenten, rascher beim Erhitzen. Es krystallisiert aus Alkohol in gelbbraunen Blättchen und schmilzt bei $16^{\circ}$.

$0.1564 \mathrm{~g}$ Sbst.: $0.4868 \mathrm{~g} \mathrm{CO}_{2}, 0.0705 \mathrm{~g} \mathrm{H}_{2} \mathrm{O} .-0.1292 \mathrm{~g}$ Sbst.: $11.8 \mathrm{cem}$ N $\left(18^{0}, 712 \mathrm{~mm}\right)$.

$$
\begin{aligned}
& \mathrm{C}_{19} \mathrm{H}_{14} \mathrm{~N}_{2} \text {. Ber. C 84.9, H 5.0, N 9.9. } \\
& \text { Gef. \ 85.1, } \$ 5.0, \gg 10.0 \text {. }
\end{aligned}
$$

Das Produkt ist wenig löslich in Wasser, Ligroin und $\vec{A}$ ther, löslicher in Alkohol, Benzol, Toluol. Dureh Săuren wird es in die Koniponenten gespalten.

1) B. 20, 1547 [1887]. 
Zur Darstellung des Oxims wurde die methylalkoholische Lösung dęAldehyds mit der berechneten Menge Hydroxylaninlösung (aus Chlorhydrat + Natriummethylat) ca. 21/2 Stunden am Rückflußkühler gekocht. Es scheided sich gelbe Nadeln aus, die durch Krystallisation aus Methylalkohol gereinigt wurden. Schmp. $247^{\circ}$ unter Zersetzung.

$0.1505 \mathrm{~g}$ Sbst.: $0.4165 \mathrm{~g} \mathrm{CO}_{2}, 0.0616 \mathrm{~g} \mathrm{H}_{2} \mathrm{O} .-0.1270 \mathrm{~g}$ Sbst.: $14.9 \mathrm{ccm}$. $\mathrm{N}\left(19^{\circ}, 740 \mathrm{~mm}\right)$.

$$
\begin{aligned}
& \mathrm{C}_{14} \mathrm{H}_{10} \mathrm{~N}_{2} \mathrm{O} \text {. Ber. C } 75.7, \mathrm{H} 4.5, \mathrm{~N} 12.6 . \\
& \text { Gef. } \$ 75.5,>4.6, \times 12.7 .
\end{aligned}
$$

Mit Salzsăare gibt das Oxim ein in gelbroten Nadelı krystallisierendes. Chlorhydrat, das sich bei $252^{\circ}$ zersetzt.

\section{Hydrolytische Spaltung der Chinaldin- und Picolin- Kondensationsprodukte.}

Die grünen Nädelcben des Chinaldinjodäthylat-Kondensationsproduktes lösen sich in verdünnter Salzsäure in der Kälte augenblicklich mit gelblicher Farbe auf. Setzt man dieser salzsauren Lösung sofort ${ }^{1}$ ) die berechnete Menge Phenylhydrazin zu ( 1 Gew.-Teil anf 4 Gew.Teile des Azomethins), so scheidet sich das Hydrazon alsbald als roter, dicker Niederschlag ab. In den davon abgetrennten Mutterlaugen findet sich neben weiteren geringen Mengen des Hydrazons das zweite Spaltungsprodukt, Dimethyl-phenylendiamin, das durcb die Methylenblau- und Mercurinitrat-Reaktion leicht nachgewiesen werden kann. Das rote Hydrazon wird durch Krystallisation aus Wasser oder Alkohol gereinigt. Aus Wasser bilden sicb rote, derbe Nadeln, die ein Molekül Krystallwasser enthalten.

$0.1749 \mathrm{~g}$ Sbst.: $0.3307 \mathrm{~g} \mathrm{CO}_{2}, 0.0751 \mathrm{~g} \mathrm{H}_{2} \mathrm{O} .-0.1607 \mathrm{~g}$ Sbst.: $0.0910 \mathrm{~g}$ Ag J.

$$
\begin{aligned}
& \mathrm{C}_{18} \mathrm{H}_{18} \mathrm{~N}_{3} \mathrm{~J}, \mathrm{H}_{2} \mathrm{O} \text {. Ber. C 51.3, H 4.7, J 30.2. } \\
& \text { Gef. 》51.6, } 4.8 \text {, 30.6. }
\end{aligned}
$$

Im Vakuum und bei $100^{\circ}$ verliert die Substanz das Krystallwasser. Sie schmilzt dann ziemlich scharf bei $245^{\circ}$.

$0.1516 \mathrm{~g}$ Sbst.: $0.3000 \mathrm{~g} \mathrm{CO}_{2}, 0.0638 \mathrm{~g} \mathrm{H}_{3} \mathrm{O} .-0.1266 \mathrm{~g}$ Sbst.: $11.8 \mathrm{ccm}$ N $\left(20^{\circ}, 719 \mathrm{~mm}\right)$.

$$
\begin{aligned}
& \mathrm{C}_{18} \mathrm{H}_{18} \mathrm{~N}_{3} \text { J. Ber. C 53.6, H 4.5, N 10.4. } \\
& \text { Gef. } \$ 54.0, \quad 4.7 \text {, } 10.3 \text {. }
\end{aligned}
$$

1) Nach kurzem Stehen, rascher beim leichten Erwärmen tritt cine Trübung der Lösung ein, indem sich in sehr geringen Mengen ein voluminöser braunschwarzer Niederschlag bildet. Unterwirft man die Flüssigkeit der Wasserdampfdestillation, so destilliert anfänglich Jod über. Die in ihrem Wesen noch nicht aufgeklärte Zersetzung ist aber nur teilweise, denn auch nach längerer Zeit läßt sich das Halogenalkylat des Aldehyds durch Phenylhydrazin noch ausfällen. 
Das Hydrazon löst sich in konzentrierter Scbwefelsäure und gibt darin mit Oxydationsmitteln eine blaue Färbung, die beim Verdünnen mit Wasser in orange umschlägt. (Bülowsche Reaktion.)

In gleicher Weise wurde das Jodmethylat des Dimethylaminoanils des Pyridyl-2-methanals mit Salzsäure und Phenylhydrazin behandelt. Die Spaltung ist auch hier momentan, dagegen verläuft die Kondensation zum Hydrazon langsamer und wird vorteilhaft durch Kocben gefördert. Das Hydrazon krystallisiert aus heißem Wasser in prachtrollen orangegelben Nadeln mit bläulichem Oberflächenschimmer. Es schmilzt bei $244^{\circ}$ unter langsamer Zersetzung und gibt ebenfalls die Bülowsche Reaktion. Die Ausbeute ist beinahe quantitativ. Die Substanz entbält zwei Moleküle Krystallwasser, die bei $100^{\circ}$ abgegeben werden.

$1.3146 \mathrm{~g}$ Sbst. verloren $0.1228 \mathrm{~g} \mathrm{H}_{2} \mathrm{O}$.

$\mathrm{C}_{13} \mathrm{H}_{14} \mathrm{~N}_{3} \mathrm{~J}, 2 \mathrm{H}_{2} \mathrm{O}$. Ber. $\mathrm{H}_{2} \mathrm{O}$ 9.6. Gef. $\mathrm{H}_{2} \mathrm{O}$ 9.3.

$0.1260 \mathrm{~g}$ Sbst.: $14.2 \mathrm{ccm} \mathrm{N}\left(20^{\circ}, 714 \mathrm{~mm}\right) .-0.1187 \mathrm{~g}$ Sbst.: $0.0806 \mathrm{~g}$ Ag J.

$$
\begin{array}{ll}
\mathrm{C}_{13} \mathrm{H}_{14} \mathrm{~N}_{3} \text { J. } & \text { Ber. N 12.4, J 37.4. } \\
& \text { Gef. "12.3, "36.7. }
\end{array}
$$

Genf, Organisches Laboratorium der Universität.

\section{A. Hantzsch:}

\section{Berichtigungen zu der Arbeit von K. Auwers "Über die Absorptions- und Refraktions-Methoder.}

(Eingegangen am 31. Mai 1912.)

Die kürzlich unter diesem Titel erschienenen, sebr ausführlichen Erörterungen und Diskussionen des Hrn. A u wers ${ }^{1}$ ) veranlassen micb, wenigstens auf einige der ron ihm kritisierten Punkte nochmals kurz einzugehen.

1. Hr. A uwers hält meinem Einwurf gegenüber daran fest, ^daß die Kohlenwasserstoffe der Terpenreibe bei der gewöhnlichen Destillation nicht in merklicher Weise verändert werden*. Hierzu wird mir auf meine Anfrage rom besten Kenner dieses Gebietes, O. Wallach, auf den sich Hr. A uwers hierbei beziehen zu dürfen glaubt, freundlichst mitgeteilt, $» \mathbf{d a}$ er $\mathbf{z}$ war den Siedepunkt der Terpene bei gewöhnlichem Druck anzugeben für zweckmäßig bält, zur Reingewinnung und Bestimmung der Konstanten aber von jeher im Va-

1) B. $4 \tilde{5}, 963-976$ [1912]. 